\title{
FORENSIC IDENTIFICATION OF COMPRESSIVELY SENSED SIGNALS
}

\author{
Xiaoyu Chu, Matthew C. Stamm, and K. J. Ray Liu
}

Dept. of Electrical and Computer Engineering, University of Maryland, College Park

\begin{abstract}
Identifying a signal's origin and how it was acquired is an important problem for digital forensics. Recently, compressive sensing has achieved substantial attention due to its ability to accurately acquire sparse signals at rates below the Nyquist rate. The increased popularity of this signal acquisition technique gives rise to a new forensic problem: is it possible to distinguish signals that have been compressively sensed from traditionally sampled ones? In our previous work, we addressed this problem of differentiating between traditionally acquired and compressively sensed images. In this paper, we examine the problem of distinguishing traditionally sampled signals from compressively sensed ones for a broader class of signals. We categorize those compressive sensing applicable signals into two cases: sparse signals with noise and nearly sparse signals. For each category, we discuss the traces left in a signal by compressive sensing and propose a corresponding detection scheme. Experimental results show that both of our proposed detection schemes can be effectively used to distinguish compressively sensed signals from traditionally sensed signals.
\end{abstract}

Index Terms - Compressive Sensing, Identification Forensics, (Nearly) Sparse Signals.

\section{INTRODUCTION}

Determining a signal's origin, how it was acquired, and how it was stored is an important set of forensic problems. Most prior work on signal acquisition forensics is targeted towards digital images. Forensic techniques have been designed to determine an image's source camera using noise introduced by sensor imperfections [1] and unique traces left by a camera's internal processing [2]. Other forensic techniques are able to identify the source coder used when compressing an image for storage [3]. While these techniques have proven to be very successful, they are only capable of operating on digital images. As a result, there is a growing need for acquisition forensic techniques capable of operating on a broader class of signals

Recently, a new signal acquisition technique known as compressive sensing has been developed [4]. While traditional sensing techniques employ uniform sampling and are thus limited by the Nyquist rate, compressive sensing techniques enable sparse signals to be sampled at a much lower rate. Rather than uniformly sampling the signal, compressive techniques obtain a series of of measurements by projecting the signal onto a special set of basis vectors. As long as enough number of measurements are collected, the original signal can be reconstructedby solving a linear minimization problem.

Because compressive sensing allows signals to be acquired with far fewer measurements that uniform sampling, it has been adapted for use in a variety of applications. One well known application of compressive sensing is the single pixel camera [5]. This camera operates by using an array of mirrors to perform a pseudorandom projection of the scene onto a single pixel sensor several times. Compressive sensing has been successfully applied to variety of other imaging applications such as magnetic resonance imaging [6], photo-acoustics [7], and astronomic images [8]. While imaging is an important application of compressive sensing, it is certainly not the only one. Compressive sensing has also been applied to sparse electronic and electromagnetic signals such as electrocardiography [9] and radar [10] [11] [12]. Compressive sensing has even seen applications in sparse network events such as the on and

Email: \{cxygrace,mcstamm,kjrliu\}@umd.edu.

This work is supported in part by AFOSR grant FA95500910179. off status of a channel in cognitive radio [13] and the occurrence of events in sensor networks [14].

In the face of such widespread use of compressive sensing, the following question naturally arises: can a signal acquired using compressive sensing be forensically distinguished from one that has been traditionally sampled? In previous work, we addressed the problem of identifying images that had been compressively sensed [15]. In this paper, we propose techniques to forensically identify compressive sensing that can be used on a broader class of signals.

The rest of the paper is organized as follows: In section II, system models are presented, where we generalize those observed signals into two basic and simple categories: sparse signals with noise and nearly sparse signals. Section III and section IV analysis the fingerprint and propose the detection scheme for each of the model, respectively. Simulation results in section $\mathrm{V}$ show that both detection schemes yield perfect detection - achieve probability of detection of $100 \%$ with probability of false alarm of $0 \%$ - when the ratio of the number of compressive measurements to the signal length is less than 0.92, which is normally a reasonable choice for compressive sensing. Section VI concludes our work.

\section{SYSTEM MODEL}

In order to reap the benefits of compressive sensing, a signal should be sparse in some domain. Theory states that if enough compressive measurements of the sparse signal are taken, the signal can be perfectly reconstructed [4]. In reality, however, virtually no signal is truly sparse. Instead, compressive sensing is applied to signals that can be well approximated as sparse. In this paper, we model these signals as either (1) a sparse signal in the presence of noise or (2) a signal that is not truly spares but is 'nearly sparse'.

There are many cases where the signals are modeled as sparse signals corrupted with noise. For example, representations on the timefrequency shift operator bases of radar signals are sparse [11]. Both [10] and [12] modeled radar signals as sparse signals corrupted with noise. For wireless sensing networks, the occurrence of events generated by sources can be modeled as sparse signals that thermal noise is added to when taking measurements in compressive sensing [14]. Additionally, cognitive radio signals can be modeled as sparse signals in the presence of noise [13].

In addition to sparse signals in noise, compressive sensing is often applied to a class of nearly sparse signals known as compressible signals [16]. Rather than consisting of a small number of impulses, the coefficients of these nearly sparse signals decay rapidly when ordered in descending order. A common example of a signal of this type is the set of discrete wavelet coefficients corresponding to a photograph of a natural scene. These have been shown to exhibit a $1 / n$ decay [17]. Other signals, such as certain classes of oscillatory signals, also have rapidly decaying Gabor coefficients [18].

In following subsections, we describe the sparse signal with noise model and the nearly sparse signal model in detail.

\section{II-A. Sparse Signals with Noise Model}

Uniformly sampling a continuous-time sparse signal $f(t), 0 \leq t \leq T$ yields a discrete signal $\mathbb{S}$ which we write in vector form. This signal $\mathbb{S}=\left[s_{1}, s_{2}, \ldots, s_{N}\right]^{T}$, which we refer to as the true signal, is an $N$ by 1 vector where $N$ is determined by Nyquist-Shannon sampling theorem. Let $K(\ll N)$ denote the number of non-zero components in S. 
Under many circumstances, the signal is corrupted by noise during sensing. When this occurs, we write the signal as

$$
x_{i}^{n q}=s_{i}+n_{i}^{n q}, \quad i=1,2, \ldots, N,
$$

where the superscript ' $n q$ ' denotes that the acquisition method is uniform sampling at the Nyquist rate and $n_{i}^{n q}, i=1,2, \ldots, N$ is zero mean i.i.d. additive measurement noise, drawn from a certain distribution $\mathcal{F}$, which is unknown. Thus the observed sparse signal with noise can be presented in matrix notation as

$$
\mathbb{X}^{n q}=\mathbb{S}+\mathbb{N}^{n q} .
$$

When compressive sensing is used to acquire the signal $\mathbb{S}$, however, each measurement is obtained by

$$
y_{i}=\phi_{i}^{T} \mathbb{S}+n_{i}^{c s}, \quad i=1,2, \ldots, M,
$$

where $\phi_{i}$ is a column vector from a sensing matrix [4] and $n_{i}^{c s}, i=$ $1,2, \ldots, M$ is i.i.d. additive measurement noise drawn from the same distribution $\mathcal{F}$ as the noise which occurs in traditional sampling. Here, the superscript ' $c s$ ' denotes that the acquisition method is compressive sensing. $M$ is the total number of compressive measurements. If the signal is sensed in a noiseless setting, it can be perfectly reconstructed as long as $M$ satisfies $M=O(K \log N)$. If it is corrupted by zero mean Gaussian noise, however, this constraint on $M$ also guarantees reconstruction error within order of $(M / \log N)^{-1}$.

The observed signal $\mathbb{X}^{c s}$ is reconstructed by solving the $l_{1}$ minimization problem

$$
\min \left|\mathbb{X}^{c s}\right|_{l 1} \text { s.t. }\left|\phi_{i}^{T} \mathbb{X}^{c s}-y_{i}\right| \leq \epsilon, i=1,2, \ldots, M,
$$

where $\epsilon$ is determined by satisfying $\left|n_{i}^{c s}\right| \leq \epsilon$.

\section{II-B. Nearly Sparse Signals Model}

In this model, let $s_{(1)} \geq s_{(2)} \geq \ldots \geq s_{(N)}$ denote the reordered components of $\mathbb{S}=\left[s_{1}, s_{2}, \ldots, s_{N}\right]^{T}$. Recall that $N$ is the length of the discrete signal acquired by Nyquist sampling. We say signal $\mathbb{S}$ obeys a power law or $1 / n$ decay if it belongs to the weak $l_{p}$ ball of radius $R$ for some $0<p<\infty$ [16]. That is, for each $1 \leq n \leq N$, the signal obeys

$$
\left|s_{(n)}\right| \leq R \cdot n^{-1 / p} .
$$

We assume that the values of the signal are drawn from a certain distribution $\mathcal{G}$, which is known. Typically, $\mathcal{G}$ may be the Laplacian or Gaussian distribution. If the signal is traditionally sensed, then $\mathbb{X}^{n q} \in \mathcal{G}^{N}$. If it has been compressively sensed, each compressive measurement is

$$
y_{i}=\phi_{i}^{T} \mathbb{X}^{n q}, \quad i=1,2, \ldots, M .
$$

where $M$ is the number of measurements. The signal is reconstructed by solving the minimization problem

$$
\min \left|\mathbb{X}^{c s}\right|_{l 1} \quad \text { s.t. } \phi_{i}^{T} \mathbb{X}^{c s}=y_{i}, i=1,2, \ldots, M,
$$

where $\mathbb{X}^{c s}$ is the compressively sensed and reconstructed signal. The number of measurements directly determines the accuracy of signal reconstruction.

\section{SPARSE SIGNALS WITH NOISE DETECTION SCHEME}

In this section, the sparse signal with noise model is studied. We begin by discussing the fingerprints left in a signal by compressive sensing. Then we propose the detection scheme accordingly.

\section{III-A. Fingerprint Analysis}

The key point of the reconstruction algorithm is to find the sparsest result that yields the same measurements when sensed. This tends to maintain the most significant coefficients. Thus, for a sparse signals in noise, the few non-noise coefficients are expected to be well preserved, while the noise coefficients are mostly thrown away during reconstruction. Because of this, there is little difference between a traditionally sensed and compressively signal in the parts of the signal corresponding to the true signal. Differences between the signals do
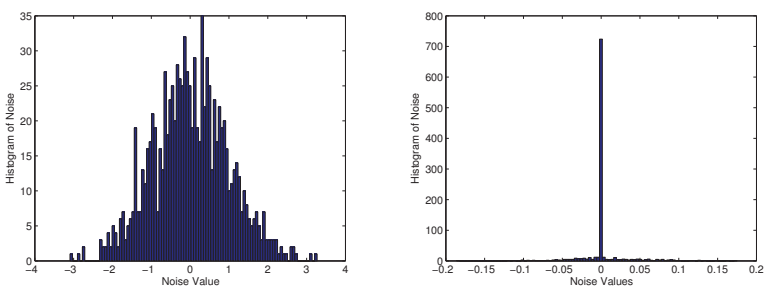

Fig. 1. Histograms of the noise extracted from the observed sparse signals with noise, acquired by traditional sensing(left), and by compressive sensing (right).

occur, however in the non-significant parts of the signal corresponding to noise.

Fig. 1 shows the noise histograms of a signal that has been traditionally sensed and compressively sensed. In this example, the signal has been corrupted by additive Gaussian noise with zero mean. We note that the noise values in the compressively sensed signal are smaller than those in traditionally sensed signals. This suggests that compressive sensing has a 'de-noising' effect. Despite this, compressively sensed signals can still be confused with traditionally sensed signals obtained in the presence of low power noise. Given the assumption that we do not know the power of the additive noise, we cannot simply differentiate between the two sensing methods by measuring a signal's noise power Instead, we exploit the fact that for most typically encountered noise distribution models, the probability that a given noise value is zero is typically zero, while the noise of compressively sensed signal has much more zero components. Fig. 1 shows an example of such difference.

\section{III-B. Forensic Detection Scheme}

Based on the fingerprint we presented in previous subsection, where compressive sensing gathers most of the non-significant coefficients to zero, we first give the intuition of our detection scheme.

Let $\mathcal{N}$ be a random variable representing a component in the noise part $\mathbb{N}$ of the observed signal $\mathbb{X}$. Denote $w_{1}$ and $w_{2}$ as the set of signals that have been traditionally sensed and compressively sensed respectively. Our problem can be formulated as determining which class $\left(w_{1}\right.$ or $\left.w_{2}\right)$ a signal $\mathbb{X}$ belongs to. Based on our observation that compressive sensing sets many of the noise values to zero, we assume

$$
\mathbb{P}\left[\mathcal{N}=0 \mid \mathbb{X} \in w_{1}\right] \ll \mathbb{P}\left[\mathcal{N}=0 \mid \mathbb{X} \in w_{2}\right],
$$

and we can take advantage of this disparity to design our detection scheme.

Given a sequence of observed sparse and noisy signal $\mathbb{X}$, our detection scheme is as follows:

1) Exact the noise part $\mathbb{N}$ from the observed signal $\mathbb{X}$. We assume the noise and true signal part can be well separated.

2) Obtain the histogram $h(k)$ of the noise $\mathbb{N}$. We gather the signal values into 100 bins in our simulation.

3) Calculate the zero coefficient ratio $\mathbb{P}_{0}$ by normalizing $h(k)$ :

$$
h^{(\text {norm })}(k)=\frac{h(k)}{\sum_{k} h(k)}
$$

and extracting the magnitude of the bin which contains the zero value.

4) Classify the signal according to the decision rule:

$$
\begin{aligned}
& \text { If } \mathbb{P}_{0}<\tau_{1}, \text { we decide } \mathbb{X} \in w_{1} \\
& \text { Otherwise, we decide } \mathbb{X} \in w_{2},
\end{aligned}
$$

where $\tau_{1}$ is some small positive constant.

\section{NEARLY SPARSE SIGNALS DETECTION SCHEME}

In this section, we first discuss the fingerprint of compressive sensing on nearly sparse signals. Then our detection scheme is proposed accordingly. 

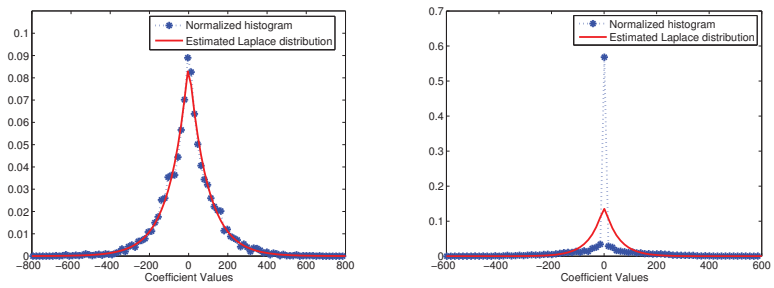

Fig. 2. Histograms of the nearly sparse signals acquired by traditional sensing (left), and by compressive sensing (right). Together with the estimated Laplacian distributions.

\section{IV-A. Fingerprint Analysis}

Since the reconstruction of compressive sensing tends to preserve coefficients with large values and sets small values to zero in order to obtain a sparse result, compressively sensed signals are likely to have many more zero valued coefficients than traditionally sensed signals. Fig. 2 shows an example of a nearly sparse signal whose coefficients are distributed according to the Laplace distribution. From the right figure, we can see that the signal that been compressively sensed deviates from the original model in the sense of its histogram. Specifically, this signal has much more kurtosis compared with the original model $\mathcal{G}$. If a model of the signal distribution is known, this fact makes it possible to distinguish a compressively sensed signal form a uniformly sampled one by measuring the distance between the signal's actual distribution and its model distribution.

Thus, in order to distinguish compressively sensed signals from traditionally sensed ones, we measure the distance between the observed the histogram of the signal and the known model distribution. Since compressive sensing sets many non-significant components to zero, we propose a modified KL-divergence in our detection scheme to emphasize this difference at zero. Specifically, we weight the distribution difference at zero the most and decrease the weight rapidly as the absolute value increasing. Then those signals, whose histograms differ the estimated distributions most at zero and the differences become smaller when the absolute value increases, will yield the highest modified KL-divergence among all signals having histograms deviate from the known distribution model $\mathcal{G}$.

\section{IV-B. Forensic Detection Scheme}

We first give the expression of the discrete form $f_{G}(k), k \in \mathbb{K}$ of a given distribution function $G(x), x \in \mathbb{R}_{G}$, where $\mathbb{K}$ is a uniform separation of $\mathbb{R}_{G}$, and each element in $\mathbb{K}$ is the center of a certain section. Let $d$ denote the length of each separated section. Then

$$
f_{G}(k)=G(k+d / 2)-G(k-d / 2), \quad k \in \mathbb{K} .
$$

And our modified KL-divergence is defined in discrete form as follows: Given distribution function $P(x)$ and $Q(x)$, let $f_{P}(k), k \in \mathbb{K}$ and $f_{Q}(k), k \in \mathbb{K}$ denote the discrete forms of $P(x)$ and $Q(x)$ respectively. Note that the separations $\mathbb{K}$ are the same for both distributions. We choose the weight distribution function as

$$
W(x)=\frac{1}{2} e^{-|x|}
$$

to emphasize the importance of the difference at zero point and reduce the effect of large coefficient disparities. Let $f_{W}(k), k \in \mathbb{K}$ denote its discrete form, which is

$$
f_{W}(k)= \begin{cases}1-e^{-\frac{d}{2}} \cosh (k) & \text { when } k+\frac{d}{2}>0 \& k-\frac{d}{2}<0 \\ e^{-|k|} \sinh \left(\frac{d}{2}\right) & \text { otherwise. }\end{cases}
$$

Then the modified KL-divergence can be written as

$$
D_{m K L}(P \| Q)=\sum_{k \in \mathbb{K}} f_{W}(k) \cdot\left|\ln \frac{f_{P}(k)}{f_{Q}(k)}\right| .
$$

We take the absolute value of the logarithmic term to maintain the symmetry of the divergence between $P(x)$ and $Q(x)$.

Given a sequence of observed nearly sparse signal $\mathbb{X}$, our detection scheme is as follows:

1) Use maximum likelihood estimation to estimate the parameters of the known distribution model $\mathcal{G}$ based on the statistics of the signal, and obtain the estimated distribution function $Q(x)$.

2) Obtain and normalize the histogram of the signal using equation (9) to get $f_{P}(k), k \in \mathbb{K}$. Record the locations of the bins in vector form $\mathbb{K}$. $d$ is also known by calculating the distance between two adjacent bins.

3) Calculate the discrete forms of $Q(x)$ and $W(x)$ using equation (10) and (12) respectively. Further normalize these two using equation (9) to finally get $f_{Q}(k), k \in \mathbb{K}$ and $f_{W}(k), k \in \mathbb{K}$.

4) Calculate the modified KL-divergence described in (13) where the summation is taken over $k \in \mathbb{K}$.

5) We also use $w_{1}$ and $w_{2}$ to denote whether this signal has been traditionally sensed or compressively sensed respectively, the decision rule is

If $D_{m K L D}(P \| Q)<\tau_{2}$, we decide $\mathbb{X} \in w_{1}$

Otherwise, we decide $\mathbb{X} \in w_{2}$,

where $\tau_{2}$ is some positive constant determined according to the signal model $\mathcal{G}$ we assumed.

\section{SIMULATIONS AND RESULTS}

To test the effectiveness of our detection scheme on signals that can be modeled as sparse signals with noise, we first simulate a sparse signal with length $N=1000$ and $K=80$ non-zero coefficients drawn from $\mathcal{N}(10,1)$. We use Basis Pursuit De-Noising [19] as our reconstruction algorithm.

In our first set of simulations, we studied the detection scheme performance under different $M / N$, which is the ratio between number of measurements in compressive sensing $M$ and the total length of the signal $N$. Additive noise values are drawn from $\mathcal{N}(0,3)$. We tested variety of $M / N$, and for each value, we repeat the simulation 200 times to obtain the Receiver Operating Characteristic (ROC) curve. Results show that our detection scheme yields perfect detection, i.e., has probability of detection of $100 \%$ with probability of false alarm of $0 \%$, when $M / N<0.94$.

The left figure in Fig. 3 plots some of the ROC curves with extreme cases of $M / N$ from our first set of simulations to show the trend of the change. Specifically, it shows that the performance of our detection scheme is better with lower $M / N$. This is because as $M$ increases, the reconstruction will yield sparser result, as it is in equation (4). Thus, the lower $M / N$ is, the sparser of the reconstructed signal. Then we will have higher zero coefficient ratio in noise part of the compressively sensed signal, which increases the difference between these two schemes in terms of zero coefficient ratio in noise, and our detection scheme gets better.

Then we further exploit the effect of the noise model and its power on our detection scheme, the right and the center figures in Fig. 3 respectively. Since we have shown in the above paragraph that our detection scheme gives perfect detection for commonly used $M / N$ 's. In order to show the trend of the ROC curves changes with different conditions, we use rather extreme cases on $M / N$ for the following two simulations. First, we still use zero-mean Gaussian as the noise model but with different power, which is just its variance in this case. $M / N=$ 0.99, and each ROC curve is obtained from 200 repeated simulations. The center figure in Fig. 3 shows that our detection scheme performs better with higher noise power. This is because the higher the noise power is, the lower the zero coefficient ratio of the traditionally sensed signal is, which enlarge the difference between these two schemes in terms of zero coefficient ratio, and our detection scheme gets better.

For the test on different noise model, we also take an uncommon $M / N=0.94$ just to show the change of detection performance with different noise models. We fix the noise power to be 3 , and simulate five noise models as in the right figure of Fig. 3. Again, each ROC 

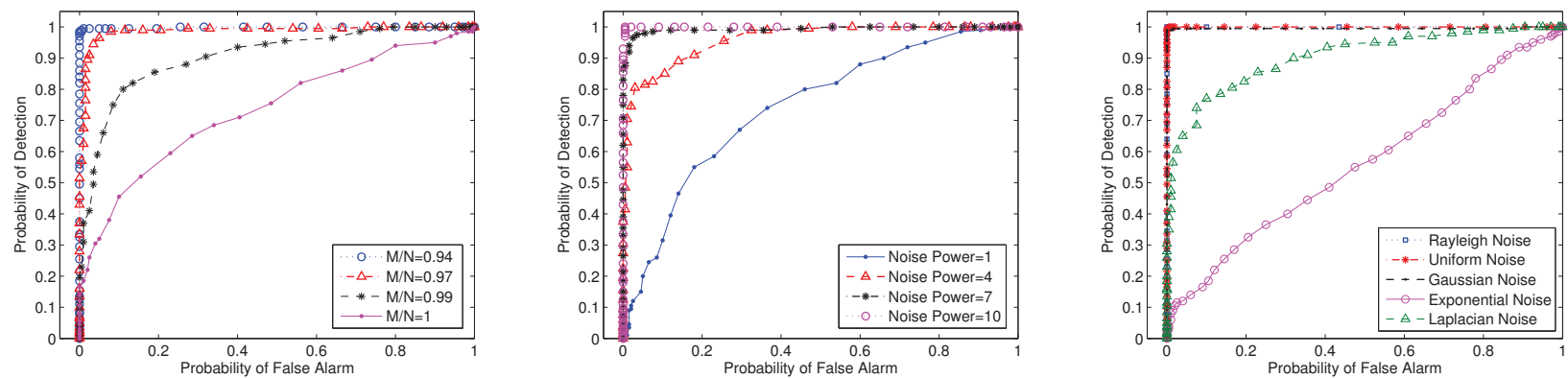

Fig. 3. ROC curves of our detection scheme for sparse signals with noise model where different $\mathrm{M} / \mathrm{N}$ (left), different noise powers (center) and different noise models (right) are used

curve is acquired from 200 repeated simulations. This result shows that Uniform and Rayleigh noise models yield comparable detection performance with Gaussian model, while we encounter some difficulty with Laplacian and Exponential models. This is because Laplacian and Exponential distributions have more kurtosis than the other three do, i.e., they have higher probability density values at zero point when powers are the same, thus the noise drawn from these model will yield higher zero coefficient ratio in traditionally sensed signals, and then make them closer to compressively sensed signals in terms of zero coefficient ratio, and consequently more difficult to distinguish. In addition, we have also tested different $M / N$ 's for Laplacian and Exponential models, and find out that our detection scheme only fails to achieve perfect detection when $M / N>0.9$ and $M / N>0.89$ for Laplacian and Exponential models respectively.

In order to test the effectiveness of our detection scheme for nearly sparse signals model, we simulate the nearly sparse signal according to a Laplace distribution, i.e., $\mathcal{G}$ is Laplacian. We test our detection scheme's performance for a variety of $M / N$, and use the results to generate a series of ROC curves. We use the Laplace distribution as the model of the nearly sparse signal because it is commonly used to model the distribution of the discrete wavelet transform coefficients of natural images [20]. We take the variance of the distribution as $2 * 10^{4}$ and set the signal length as $N=5000$. Different number of measurement $M$ is used and ROC curve is plotted for each $M$ by repeating the simulation 200 times. In all cases where $M / N<0.92$, our compressive sensing detection technique achieved perfect detection. Additionally, we observed the performance of our detection technique gets better with less measurements. This is because the reconstruction error of compressive sensing increases with smaller $M$ [16]. Thus, the less the measurements, the larger the difference between the reconstructed signal of compressive sensing and the original signal, which is the same as the traditionally sensed signal. And then makes it easier to distinguish these two by observing the divergence.

\section{CONCLUSIONS}

In this paper we studied the acquisition forensics of compressively sensed signals. We generalized the compressive sensing applicable signals into two categories: sparse signals with noise and nearly sparse signals. For the first model, we used the zero coefficient ratio of the noise to distinguish compressively sensed signals from traditionally sensed signals. Simulations show that our detection scheme yields perfect detection when $M / N<0.94$. And the performance gets better with less measurements in compressive sensing and lower ratio of zero coefficients in noise model. For the nearly sparse signals model, we proposed a modified KL-divergence as a detection criterion, and simulation shows that this modified one yields perfect detection when $M / N<0.92$.

\section{REFERENCES}

[1] M. Chen, J. Fridrich, M. Goljan, and J. Lukášs, "Determining image origin and integrity using sensor noise," IEEE Trans. Inf. Forensics Security, vol. 26, no. 2, pp. 38-48, Mar. 2009.
[2] A. Swaminathan, M. Wu, and K. J. R. Liu, "Component forensics," IEEE Trans. Sig. Proc., vol. 26, no. 2, pp. 38-48, Mar. 2009.

[3] W. S. Lin, S. K. Tjoa, H. V. Zhao, and K. J. R. Liu, "Digital image source coder forensics via intrinsic fingerprints," IEEE Trans. Inf Forensics Security, vol. 4, no. 3, pp. 460-475, Sep. 2009.

[4] D. L. Donoho, "Compressed sensing," IEEE Trans. Inf. Theory, vol 52, no. 4, pp. 1289-1306, Apr. 2006.

[5] M. F. Duarte, M. A. Davenport, D. Takhar, J. N. Laska, T. Sun, K. F. Kelly, and R. G. Baraniuk, "Single-pixel imaging via compressive sampling," IEEE Sig. Proc. Mag., vol. 25, no. 2, pp. 83-91, Mar. 2008.

[6] M. Lustig, D. L. Donoho, J. M. Santos, and J. M. Pauly, "Compressed sensing mri," IEEE Sig. Proc. Mag., vol. 25, no. 2, pp. 72 - 82, Mar. 2008.

[7] J. Provost and F. Lesage, "The application of compressed sensing for photo-acoustic tomography," IEEE Trans. Medical Image, vol. 28, no. 4, pp. 585 - 594, Apr. 2009.

[8] J. Bobin, J.-L. Starck, and R. Ottensamer, "Compressed sensing in astronomy," IEEE J. Sel. Topics in Sig. Proc., vol. 2, no. 5, pp. 718 - 726, Oct. 2008.

[9] E. G. Allstot, A. Y. Chen, A. M. R. Dixon, D. Gangopadhyay, and D. J. Allstot, "Compressive sampling of ecg bio-signals: Quantization noise and sparsity considerations," Proc. IEEE BioCAS, pp. 41-44, Nov. 2010.

[10] B. Pollock and N. A. Goodman, "Detection performance of compressively sampled radar signals," Proc. IEEE RadarCon, pp. 1117 1122, May 2011

[11] M. A. Herman and T. Strohmer, "High-resolution radar via compressed sensing," IEEE Trans. Sig. Proc., vol. 57, no. 6, pp. 2275 2284, Jun. 2009

[12] J. Tian, J. Sun, Y. Zhang, N. Ahmad, and X. Su, "The effects of input signal-to-noise ratio on compressive sensing sar imaging," Proc. IEEE 2nd ICSPS, vol. 3, pp. V3-533 - V3-537, Jul. 2010.

[13] Z. Tian and G. B. Giannakis, "Compressed sensing for wideband cognitive radios," Proc. IEEE ICASSP, vol. 4, pp. IV-1357 - IV1360, Apr. 2007.

[14] J. Meng, H. Li, and Z. Han, "Sparse event detection in wireless sensor networks using compressive sensing," Proc. IEEE 43rd Annual CISS, pp. $181-185$, Mar. 2009

[15] X. Chu, M. C. Stamm, W. S. Lin, and K. J. Ray Liu, "Forensic identification of compressively sensed images," Proc. IEEE ICASSP, Mar. 2012.

[16] E. J. Candes and T. Tao, "Near-optimal signal recovery from random projections: Universal encoding strategies?," IEEE Trans. Inf. Theory, vol. 52, no. 12, pp. 5406 - 5425, Dec. 2006.

[17] S. Mallat, "A wavelet tour of signal processing," San Diego, CA: Academic, 1998

[18] H. G. Feichtinger, "Atomic characterizations of modulation spaces through gabor-type representations," Rocky Mountain J. Math, vol. 19, no. 1, pp. 113 - 125, 1989.

[19] S. S. Chen, D. L. Donoho, and M. A. Saunders, "Atomic decomposition by basis pursuit," SIAM J. Scientific Comput., vol. 20, no. 1, pp. 33-61, 1999.

[20] M. C. Stamm and K. J. R. Liu, "Anti-forensics of digital image compression," IEEE Trans. Inf. Forensics Security, vol. 6, no. 3, pp. 1050-1065, Sep. 2011. 